\title{
Multi-Point Fibre Optic Hot-Spot Network Integrated Into a High Power Transformer
}

\author{
A. B. Lobo Ribeiro ${ }^{*}$, N. Eira ${ }^{\mathrm{b}}$, J. M. Sousa, P. T. Guerreiro and J. A. R. Salcedo \\ Multiwave Photonics S.A., R. Eng. Frederico Ulrich 2650, 4470-605 Moreira da Maia, Portugal; \\ ${ }^{a}$ University Fernando Pessoa, R. Carlos da Maia 296, 4200-150 Porto, Portugal; \\ ${ }^{\mathrm{b}}$ DEEC, Fac. Eng. University of Porto, R. Dr. Roberto Frias, 4050-123 Porto, Portugal.
}

\begin{abstract}
A multi-point fibre optic temperature sensor network integrated inside a power transformer for continuous monitoring of hot-spots on windings, cellulose insulations and oil, is demonstrated and tested. The temperature sensors are based on proprietary encapsulated fibre Bragg grating (FBG) sensors and the optical interrogation unit uses a special designed narrowband high power broadband fibre source. The fibre optic sensing network is integrated into a 440 MVA power transformer having 12 temperature sensing points, distributed over several physical locations inside de transformer (windings, cellulose insulators, magnetic circuit and cooling oil entrance and exit).
\end{abstract}

Keywords: fibre Bragg grating, fibre optic sensor, temperature measurement, power transformer.

\section{INTRODUCTION}

Transformers area among the most vital items of equipment in power systems networks, and the consequences of an unexpected failure can cause high financial damage for any energy provider. Loading capability of power transformers is limited mainly by winding temperature. During its entire operation time, a power transformer has to withstand numerous stresses of a thermal, electrical and mechanical nature [1], originating an increasing degradation of the cellulose insulation, which undergoes a de-polymerization process. The mechanical properties of the paper (tensile strength and elasticity) degrade, and the paper is not capable of withstanding short circuit forces and even normal vibrations, defining irreversibly by this way, the transformer end of life [2]. Oil-filled power transformers have and additional risk when operating at high temperature, due to the residual water trapped in paper that can reach bubbling conditions. These water vapour bubbles may move with the oil flow, or get trapped in the winding, and in both cases create a threat for insulation breakdown [2]. This is why the so-called hot-spot temperature is of great importance for the transformer owners, and the direct measurement of winding temperature is necessary [3].

There are a number of thermal models for estimating the hot-spot temperature behaviour, which uses the temperature measurements obtained outside or inside the transformer by using (a) thermocouple devices or (2) fibre optic sensors (single-point sensor or by fully distributed sensor). The thermocouple device cannot be used in the winding, but rather at the cooling oil at the bottom and at the top of the transformer, and also suffer from electromagnetic noise interference. The distributed temperature fibre optic sensor, mainly based on Raman scattering effect, although having enough temperature accuracy for power transformers, they have also a poor spatial resolution $\left( \pm 1^{\circ} \mathrm{C} / \mathrm{m}\right)$, long acquisition time and higher cost. On the other end, single-point fibre optic sensors available on the market, are based on solid-state semiconductor device (GaAs based) glued at the tip of a $200 / 230 \mu \mathrm{m}$ optical fibre, with accuracies between $\pm 1.5^{\circ} \mathrm{C}$ and $\pm 2^{\circ} \mathrm{C}$.

In this paper, the authors demonstrate a real test application of all fibre Bragg grating (FBG) temperature-sensing network embedded inside a $440 \mathrm{MVA}$ power transformer. The temperature change, and consequently, the Bragg wavelength shift of each FBG sensor, is measured using a modified version of a passive all-fibre demodulation scheme previously described by one of the authors [4]. In order to have a long optical sensor fibre link capability $(\sim 1000 \mathrm{~m})$ without sacrificing the signal-to-noise ratio of the sensors a proprietary narrowband high power ASE fibre source was fabricated.

\footnotetext{
*alobo@multiwavephotonics.com; phone +351 22940 8260; fax +351 22940 8261; www.multiwavephotonics.com
}

Third European Workshop on Optical Fibre Sensors, Antonello Cutolo, Brian Culshaw, José Miguel López-Higuera, Eds., Proceedings of SPIE Vol. 6619,66193V, (2007) $\cdot 0277-786 X / 07 / \$ 18 \cdot$ doi: 10.1117/12.738780 


\section{SYSTEM DESCRIPTION}

The configuration of the multi-point fibre optic sensor (MPOS) unit is shown schematically in Fig.1. A narrowband and spectrally flat ASE fibre optic source (see Fig.2), emitting at central wavelength of $1550 \mathrm{~nm}$ with $\sim 55 \mathrm{~mW}$ output optical power and $\sim 7 \mathrm{~nm}$ spectral width (FWHM), was used to illuminate the FBG sensing network in a star-topology which is deployed inside the power transformer.

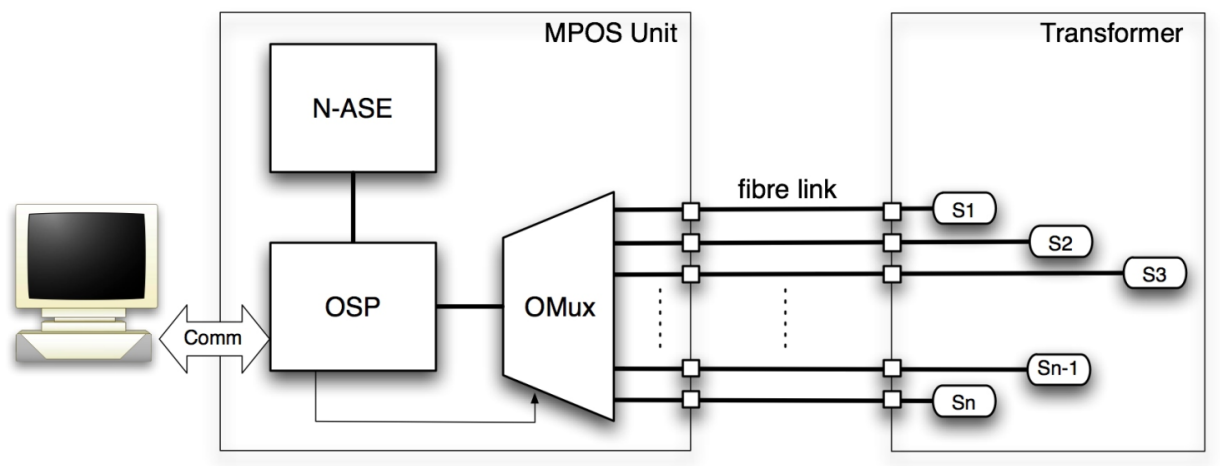

Fig. 1. Schematic diagram of the multi-point fibre optic sensor unit. (N-ASE: narrowband ASE fibre optic source, OSP: optical signal processing module, OMux: addressable optical multiplexer, Sn: fibre optic sensor number $n$ )

The optical signal-processing (OSP) module is based on a modified version of the demodulation scheme described in reference [4]. This OSP module also integrates all optical photo-detection, the 13-bit Analog-to-Digital (ADC) conversion, microcontroller and RS232/RS485 communication interface for external PC unit control and graphical user interface. The presented module has also the option to communicate with a PC via USB and/or GSM interface. This OSP module also communicates with the addressable optical multiplexer (OMux) module through an embedded programming algorithm, which digitally controls independently the optical port for each FBG sensor.

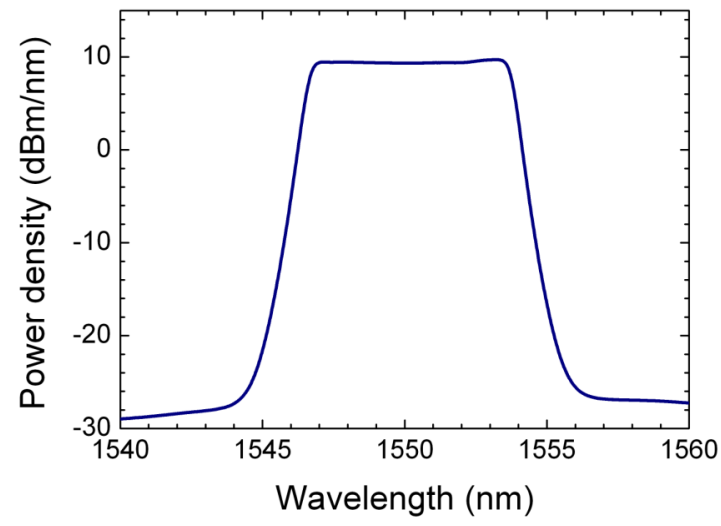

Fig. 2. Emission spectra of the narrowband ASE fibre source [5].

All the FBG sensors have an identical nominal Bragg wavelength of $1550 \mathrm{~nm}$ at room temperature, with a reflectivity around 98\%, wavelength bandwidths of 0.2 to $0.1 \mathrm{~nm}$ and a physical length of $10 \mathrm{~mm}$. The FBG sensors where encapsulated in a uniform size protective tubing (special quartz capillary and Teflon tubes), which ensure full protection of tip against mechanical stress and potential transformer oil damage. The fibre sensor cable inside the transformer is passed to the outside through a tank wall mounted feed-through vaccum channel, which avoids oil leakage, and is terminated with a FC/APC fibre optic connector/adapter. For the experimental tests, we have used a two combined fibre optic cables between transformer and MPOS unit, both of $25 \mathrm{~m}$ in length and with 6 singlemode fibre optic link each, but the system is capable of reading the signals at longer optical link distances $(\sim 1000 \mathrm{~m})$. 
Figure 3 shows a photo of the developed MPOS unit with capability to multiplex 12 FBG sensors (with expanding capability option up to 36 sensors). The main characteristics of this sensing unit are: temperature accuracy of $\pm 1^{\circ} \mathrm{C}$ and resolution of $0.1^{\circ} \mathrm{C}$ over the temperature operating range $-25^{\circ} \mathrm{C}$ to $+250^{\circ} \mathrm{C}$; maximum switching channel frequency of $50 \mathrm{~Hz}$ and a sampling sensor rate of $150 \mathrm{~Hz}$ (limited only by the photodetection electronics and for this sensing application).

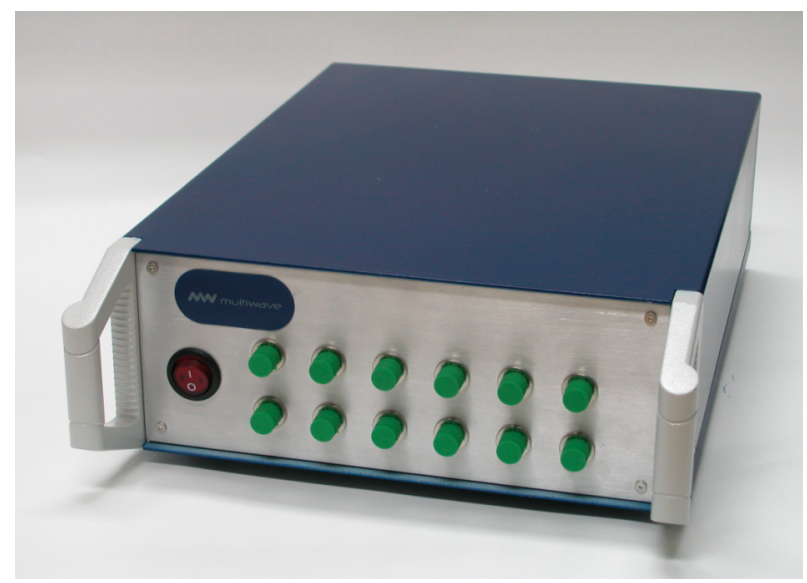

Fig. 3. Photo of the MPOS unit.

The LabView ${ }^{\mathrm{TM}}$ software package is used to perform real-time data acquisition, signal processing, network communication control and graphical user interface (GUI). Configuration and data files are saved to the user's PC, for later data analysis and temperature history record. Figure 4 shows the GUI implemented for the present sensing system.

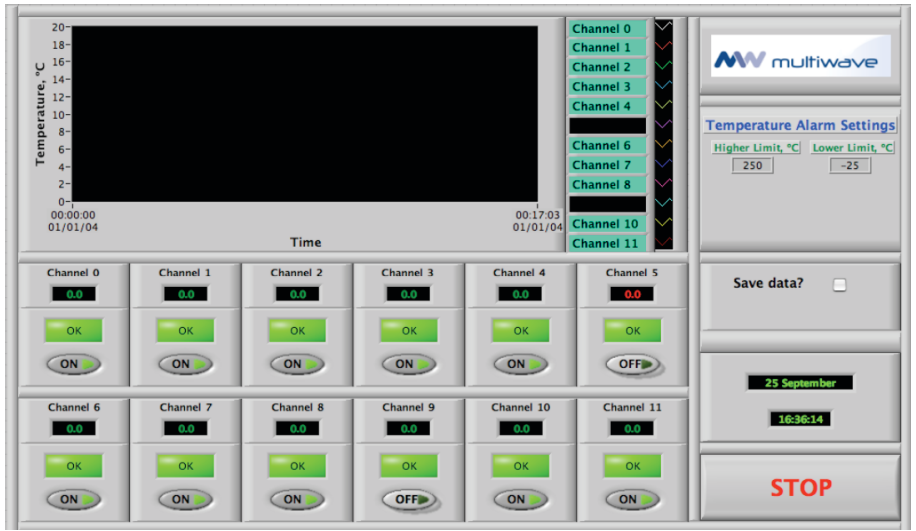

Fig. 4. The graphical user interface of the MPOS unit.

\section{RESULTS AND DISCUSSION}

The results of the temperature monitoring obtained during a laboratory load test of the 440 MVA power transformer, are presented in Figure 5. The fibre optic sensors numbered 429 and 411 are mounted in direct contact with the lower and higher voltage bars of the transformer, respectively, and the sensor 404 is glued inside the deflector tube of the high voltage cooper bar. The other sensors numbered 426, 427, 428 are distributed in different locations of the windings; the sensors 418 and 414 are located in the magnetic circuit; the sensors numbered 412, 413, 409 and 403 are glued at different positions of the oil-tank wall. The power transformer was subjected to a $10 \%$ overload condition (10\% above nominal current) at the ambient temperature of $22^{\circ} \mathrm{C}$.

This continuous temperature monitoring of power transformer provides a clear indication of his real status and ageing behaviour. During overloaded cycle accelerated ageing has to be minimized and with these on-line data, damages can be avoided. It should be mentioned that a hot-spot temperature of $120^{\circ} \mathrm{C}$, which is admissible according to the standard IEC 
60354 , causes an ageing rate factor of 12 , and thus its obvious that continuous transformer monitoring of ageing is highly recommended.

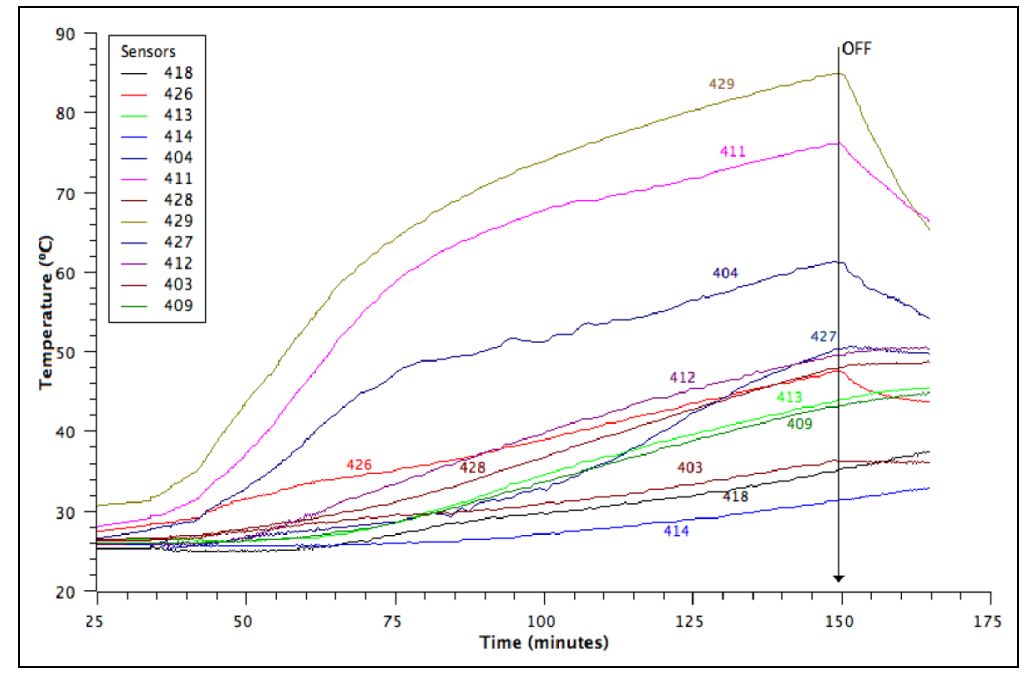

Fig. 5. Hot-spot temperature measured by the MOPS unit during a heating cycle of the 440 MVA power transformer.

It has been recently demonstrated [6], that the transformer oil has no characteristic influence on the mechanical strength of the used optical fibre type and the thermal induced decrease of the refractive index variation in the FBG does not prevent a signal processing with excellent SNR.

\section{CONCLUSIONS}

The application of fibre Bragg grating sensors for monitoring power transformer hot-spot temperatures has been demonstrated. Laboratory load tests carried on a $440 \mathrm{MVA}$ power transformer show a $0.1^{\circ} \mathrm{C}$ resolution and an overall temperature accuracy of $\pm 1^{\circ} \mathrm{C}$ to the monitoring unit developed. The sensing system has a multiplexing capability of 12 sensing probes (expandable up to 36 probes), and a real-time probe control and temperature history analysis, providing this way an on-line indication of the transformer status and ageing behaviour.

\section{ACKNOWLEDGMENTS}

The authors acknowledge the financial support from the Portuguese Innovation Agency (Agência de Inovação S.A.) under the project DEMTEC no.03/00326. The authors also wish to thank to Eng. R. Lopes and Eng. M. Silvestre of EFACEC-Energia S.A. for providing the laboratory conditions for the power transformer load test.

\section{REFERENCES}

1. T. Leibfried, “Online monitors keep transformers in service”, IEEE Comput. Appl. Power 11(3), 36-42 (1998).

2. J.N. Bérubé, J. Aubin and W. McDermid, "Transformer winding hot spot temperature determination", Fifth Annual Weidmann ACTI Technical Conf. (Albuquerque, New Mexico, Nov.27, 2006), pp.1-10, http://www.weidmann-acti.com

3. CIGRE Working Group 09 of Study Committee 12, "Direct measurement of the hot-spot temperature of transformers", CIGRE ELECTRA 129, 47-51 (1990).

4. A.B. Lobo Ribeiro, L.A. Ferreira, M. Tsvetkov and J.L. Santos, "All-fibre interrogation technique for fibre Bragg sensors using a biconical fibre filter," Electron. Lett. 17(4), 382-383 (1996).

5. Narrowband ASE fibre source (C-band), see at: http://www.multiwavephotonics.com

6. J. Teunissen, R. Merte and D. Peier, "Stability of fiber Bragg grating sensors for integration into high-voltage transformers for online monitoring”, in Proc. 15th Optical Fiber Sensors Conf. Tech. Digest, OFS 2002 (Portland, May 6-10, 2002) pp.541-544. 